ANNALES

POLONICI MATHEMATICI

$90.1(2007)$

\title{
Spectrum of certain Banach algebras and $\bar{\partial}$-problems
}

\author{
by Linus Carlsson, Urban Cegrell and \\ ANDERS FÄLLSTRÖM (Umeå)
}

\begin{abstract}
We study the spectrum of certain Banach algebras of holomorphic functions defined on a domain $\Omega$ where $\bar{\partial}$-problems with certain estimates can be solved. We show that the projection of the spectrum onto $\mathbb{C}^{n}$ equals $\bar{\Omega}$ and that the fibers over $\Omega$ are trivial. This is used to solve a corona problem in the special case where all but one generator are continuous up to the boundary.
\end{abstract}

1. Introduction and notations. In this paper we are interested in questions related to the spectrum of uniform algebras consisting of certain classes of bounded holomorphic functions on bounded domains in $\mathbb{C}^{n}$. Using techniques of Hörmander and Øvrelid among others, we first show that in the case when certain $\bar{\partial}$-equations can be solved, the projection of the spectrum onto $\mathbb{C}^{n}$ equals the closure of the domain, and that the fibers in the spectrum over interior points are trivial. In the last section, we study the fibers in the spectrum over boundary points and solve a weak form of the corona problem.

Let $\Omega$ be a domain in $\mathbb{C}^{n}$ and denote by $H(\Omega)$ the analytic functions on $\Omega, H^{\infty}(\Omega)=H(\Omega) \cap L^{\infty}(\Omega)$ and $A^{k}(\Omega)=H(\Omega) \cap C^{k}(\bar{\Omega}), 0 \leq k$ $<\infty$. The space $A^{0}(\Omega)$ will usually be denoted by $A(\Omega)$. We let $\mathcal{R}(\Omega)$ denote one of the classes $L^{\infty}(\Omega)$ or $C^{k}(\bar{\Omega}), 0 \leq k<\infty$. We denote by $\mathcal{M}$ the spectrum (= the multiplicative linear functionals) of $\mathcal{B}(\Omega)=\mathcal{R}(\Omega) \cap$ $H(\Omega)$.

If $m \in \mathcal{M}$, we denote by $\pi$ the projection $\pi m=\left(m z_{1}, \ldots, m z_{n}\right)$, and $\widetilde{\Omega}$ denotes the point evaluations in $\Omega$, i.e. $\widetilde{\Omega}=\{m \in \mathcal{M}: \pi m \in \Omega, m(f)=$ $f(\pi m)\}$. The Gelfand transform of $f \in \mathcal{B}(\Omega)$ will be denoted by $\widehat{f}(m)=m f$. By $X$ we denote the set $\overline{\widetilde{\Omega}} \backslash \widetilde{\Omega}$, and Sh is the Shilov boundary. Note that $\pi X=\partial \Omega$ and $\mathrm{Sh} \subset X$.

2000 Mathematics Subject Classification: 32A65, 32W05, 46J20.

Key words and phrases: holomorphic functions, Banach algebras, corona problem, Shilov boundary, $\bar{\partial}$-problems. 
For every natural number $0 \leq s \leq n$ we denote by $\Lambda^{s} \mathbb{C}^{n}$ the exterior product of order $s$ of $\mathbb{C}^{n}$.

Let $e_{1}, \ldots, e_{n}$ be the canonical basis in $\mathbb{C}^{n}$ and $e_{1}^{*}, \ldots, e_{n}^{*}$ the dual basis such that $\left\langle e_{j}^{*}, e_{k}\right\rangle=\delta_{j k}$. Furthermore, $e^{\beta}=e_{\beta_{1}} \wedge \cdots \wedge e_{\beta_{s}}, 1 \leq \beta_{1}<\cdots<$ $\beta_{s} \leq n$.

Define

$$
\begin{aligned}
& \mathcal{L}_{r}^{s}=\left\{\sum f_{\alpha \beta} d \bar{z}^{\alpha} \otimes e^{\beta}: f_{\alpha \beta} \in \mathcal{R}, e^{\beta} \in \Lambda^{s} \mathbb{C}^{n},|\alpha|=r,|\beta|=s\right\}, \\
& \widetilde{\mathcal{L}_{r}^{s}}=\left\{f \in \mathcal{L}_{r}^{s}: \frac{\partial^{|\gamma|}}{\partial \bar{z}^{\gamma}} f_{\alpha \beta} \in \mathcal{R}, 0 \leq|\gamma| \leq n-r\right\} .
\end{aligned}
$$

Fix a point $p=\left(p_{1}, \ldots, p_{n}\right) \in \mathbb{C}^{n}$. Define the homomorphisms $\bar{\partial}_{r}$ on $\widetilde{\mathcal{L}_{r}^{s}}$ as

$$
\bar{\partial}_{r}(u \otimes \omega)=\bar{\partial} u \otimes \omega, \text { i.e. } \bar{\partial}_{r}: \widetilde{\mathcal{L}_{r}^{s}} \rightarrow \widetilde{\mathcal{L}_{r+1}^{s}}
$$

and the homomorphisms $P_{s}: \mathcal{L}_{r}^{s} \rightarrow \mathcal{L}_{r}^{s-1}$ as follows: for $u \in \mathcal{L}_{r}^{s}$ with $u=$ $\sum_{|\beta|=s} u_{\beta} \otimes e^{\beta}$ let

$$
\left.P_{s}\left(u_{\beta} \otimes e^{\beta}\right)=\sum_{i=1}^{n}\left(z_{i}-p_{i}\right) u_{\beta} \otimes\left(e_{i}^{*}\right\lrcorner e^{\beta}\right)
$$

where

$$
\left.e_{i}^{*}\right\lrcorner e^{\beta}= \begin{cases}(-1)^{k-1} e_{\beta_{1}} \wedge \cdots \wedge \widehat{e}_{\beta_{k}} \wedge \cdots \wedge e_{\beta_{s}} & \text { if } i=\beta_{k}, \\ 0 & \text { if } i \notin \beta .\end{cases}
$$

We have the following diagram:

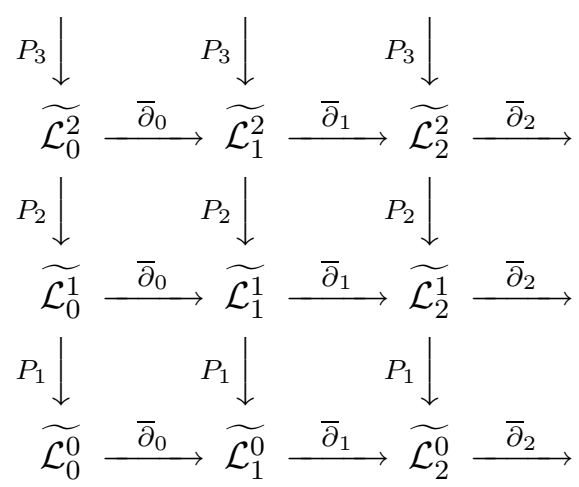

Observe that $P$ and $\bar{\partial}$ commute since $z_{j}-p_{j}$ are holomorphic. Furthermore, $P_{s}\left(\widetilde{\mathcal{L}_{r}^{s}}\right) \subset \widetilde{\mathcal{L}_{r}^{s-1}}$.

\section{The spectrum over the closure of the domain}

LEMma 2.1. Let $\Omega \subset \mathbb{C}^{n}$ be a bounded domain with the property that for every $\bar{\partial}$-closed form $\lambda \in \mathcal{R}_{(0, q)}(\Omega) \cap C_{(0, q)}^{1}(\Omega), 1 \leq q \leq n-1$, there exists 
a form $u \in \mathcal{R}_{(0, q-1)}(\Omega)$ such that $\bar{\partial} u=\lambda$. Let $p$ be a point in $\mathbb{C}^{n}$ and $f$ a function holomorphic on $\Omega$. If

$$
g_{0}^{1}(z)=\sum_{i=1}^{n} \frac{\overline{z_{i}-p_{i}}}{|z-p|^{2}} f(z) e_{i}
$$

belongs to $\widetilde{\mathcal{L}_{0}^{1}}$ then there exist $f_{i} \in \mathcal{B}, i=1, \ldots, n$, such that

$$
f(z)=\sum_{i=1}^{n}\left(z_{i}-p_{i}\right) f_{i}(z) .
$$

Proof. Note that $f(z)=P_{1}\left(g_{0}^{1}\right)(z)$ and hence the coefficients of $g_{0}^{1}$ solve the Gleason problem in $\mathcal{R}(\Omega)$ but are not necessarily holomorphic. We now inductively define

$$
g_{k}^{k+1}(z)=\frac{g_{0}^{1}(z) \wedge \bar{\partial}\left(g_{k-1}^{k}(z)\right)}{f(z)} .
$$

Subtracting the Taylor polynomial of $f$ at $p$ of degree large enough, we may assume that $g_{k}^{k+1}$ belongs to $\widetilde{\mathcal{L}_{k}^{k+1}}$. A calculation shows that

$$
P_{k} \bar{\partial}\left(\frac{g_{0}^{1}}{f} \wedge \bar{\partial} g_{k-2}^{k-1}\right)=0
$$

which implies that

$$
P_{k+1} g_{k}^{k+1}=P_{k+1}\left(\frac{g_{0}^{1}}{f} \wedge \bar{\partial} g_{k-1}^{k}\right)=\bar{\partial} g_{k-1}^{k}
$$

For $N$ large enough we get

$$
\bar{\partial} g_{N-1}^{N}=P_{N+1} g_{N}^{N+1}=0 .
$$

By the assumptions on $\Omega$ this gives us a $v_{N-2}^{N} \in \mathcal{L}_{N-2}^{N}$ such that

$$
\bar{\partial} v_{N-2}^{N}=g_{N-1}^{N} \text {. }
$$

Assuming that $v_{k-1}^{k+1} \in \mathcal{L}_{k-1}^{k+1}$ has been found such that

$$
\bar{\partial} v_{k-1}^{k+1}=g_{k}^{k+1}-P_{k+2} v_{k}^{k+2},
$$

we get, using (2.3),

$$
\bar{\partial}\left(g_{k-1}^{k}-P_{k+1} v_{k-1}^{k+1}\right)=0 .
$$

Hence, there exists a solution $v_{k-2}^{k} \in \mathcal{L}_{k-2}^{k}$ to

$$
\bar{\partial} v_{k-2}^{k}=g_{k-1}^{k}-P_{k+1} v_{k-1}^{k+1} .
$$

For $k=2$ we get

$$
g_{0}^{1}(z)-P_{2} v_{0}^{2}(z)=\sum_{i=1}^{n} f_{i}(z) e_{i} \in \mathcal{B} \otimes \mathbb{C}^{n} .
$$


Since $P_{1} g_{0}^{1}=f$, letting $P_{1}$ act on $(2.4)$ we get $f(z)=\sum_{i=1}^{n}\left(z_{i}-p_{i}\right) f_{i}(z)$ where $f_{i} \in \mathcal{B}$.

Proposition 2.2. Let $\Omega \subset \mathbb{C}^{n}$ be a bounded domain with the property that for every $\bar{\partial}$-closed form $\lambda \in \mathcal{R}_{(0, q)}(\Omega) \cap C_{(0, q)}^{1}(\Omega), 1 \leq q \leq n-1$, there exists a form $u \in \mathcal{R}_{(0, q-1)}(\Omega)$ such that $\bar{\partial} u=\lambda$. For a point $p \in \Omega$, the maximal ideal in $\mathcal{B}(\Omega)$ consisting of the functions vanishing at $p$ is generated by the functions $z_{1}-p_{1}, \ldots, z_{n}-p_{n}$.

Proof. We show this by solving the Gleason problem for $\mathcal{B}$ in $\Omega$. Let $f$ be a function in $\mathcal{B}$ vanishing at $p$. Subtracting the Taylor polynomial of $f$ at $p$ of degree large enough, we may assume that

$$
\frac{\partial^{|\alpha|+|\beta|} f}{\partial z^{\alpha} \partial \bar{z}^{\beta}}(p)=0 \quad \text { for } 0 \leq|\alpha|+|\beta| \leq M, M \text { large enough. }
$$

Defining

$$
g_{0}^{1}(z)=\sum_{i=1}^{n} \frac{\overline{z_{i}-p_{i}}}{|z-p|^{2}} f(z) e_{i}
$$

we see that $(2.5)$ implies that $g_{0}^{1}$ belongs to $\widetilde{\mathcal{L}_{0}^{1}}$. The result follows from Lemma 2.1.

Proposition 2.3. Let $\Omega \subset \mathbb{C}^{n}$ be a bounded domain with the property that for every $\bar{\partial}$-closed form $\lambda \in \mathcal{R}_{(0, q)}(\Omega) \cap C_{(0, q)}^{1}(\Omega), 1 \leq q \leq n-1$, there exists a form $u \in \mathcal{R}_{(0, q-1)}(\Omega)$ such that $\bar{\partial} u=\lambda$. Then the projection $\pi(\mathcal{M})$ of the spectrum of $\mathcal{B}(\Omega)$ onto $\mathbb{C}^{n}$ equals the closure $\bar{\Omega}$ of $\Omega$.

Proof. Suppose there exists an element $m_{0} \in \mathcal{M}$ such that $\pi m_{0}=p \notin \bar{\Omega}$. Define

$$
g_{0}^{1}(z)=\sum_{i=1}^{n} \frac{\overline{z_{i}-p_{i}}}{|z-p|^{2}} e_{i} .
$$

Since $p \notin \bar{\Omega}$ we see that $g_{0}^{1}$ belongs to $\widetilde{\mathcal{L}_{0}^{1}}$. Lemma $(2.1)$ gives functions $f_{1}, \ldots, f_{n}$ in $\mathcal{B}$ such that

$$
1=\sum_{i=1}^{n}\left(z_{i}-p_{i}\right) f_{i}(z) \quad \text { on } \Omega .
$$

Since $m_{0}$ is linear and multiplicative and since $\pi m_{0}=\left(m_{0}\left(z_{1}\right), \ldots, m_{0}\left(z_{n}\right)\right)$ $=\left(p_{1}, \ldots, p_{n}\right)$ we get

$$
1=m_{0}(1)=m_{0}\left(\sum_{i=1}^{n}\left(z_{i}-p_{i}\right) f_{i}\right)=0,
$$

which is a contradiction. 
3. Representing measures and corona type problems for $H^{\infty}(\Omega)$. In this section, we assume that $0 \in \Omega$. A representing measure for $\widetilde{0}$ is a probability measure $\mu$ such that $f(0)=\int \widehat{f} d \mu$ for all $f \in H^{\infty}(\Omega)$. We denote by $M$ the class of representing measures with support outside $\widetilde{\Omega}$; note that there is at least one element in $M$ with support in the Shilov boundary.

A closed set $K$ in $\mathcal{M}$ is called a peak set if there is an $f \in H^{\infty}(\Omega)$ with $\widehat{f}=1$ on $K$ and $|\widehat{f}|<1$ outside $K$.

Proposition 3.1. If $K$ is a peak set, then $\sup _{\mu \in M} \mu(K)=0$. On the other hand, if $K$ is a closed subset of $\mathcal{M} \backslash \pi^{-1}(\Omega)$ with $\sup _{\mu \in M} \mu(K)=0$, then there is a peak set $L$ that contains $K \cap X$.

Proof. See [1].

Proposition 3.2. Suppose that $z^{0} \in \partial \Omega$ and that $\pi^{-1}\left(z^{0}\right)$ is a peak set. Then the Shilov boundary of the algebra $\left\{\left.\widehat{g}\right|_{\pi^{-1}\left(z^{0}\right)}: g \in H^{\infty}(\Omega)\right\}$ is contained in $\mathrm{Sh} \cap \pi^{-1}\left(z^{0}\right)$.

Proof. Let $g \in H^{\infty}(\Omega)$ be given. We want to prove that

$$
\sup _{\pi m=z^{0}}|\widehat{g}(m)|=\sup _{\substack{m \in \operatorname{Sh} \\ \pi m=z^{0}}}|\widehat{g}(m)| .
$$

Since $\pi^{-1}\left(z^{0}\right)$ is a peak set, we can choose $f \in H^{\infty}(\Omega)$ with $\widehat{f}=1$ on $\pi^{-1}\left(z^{0}\right)$ and $|\widehat{f}|<1$ otherwise. For every $s \in \mathbb{N}$, choose $m_{s} \in$ Sh such that $\sup _{\pi m=z^{0}}|\widehat{g}(m)|=\widehat{\mid g f^{s}}\left(m_{s}\right) \mid$ and let $m_{0}$ be a cluster point of $\left(m_{s}\right)_{s=1}^{\infty}$. Here $\widehat{f}\left(m_{s}\right) \rightarrow 1$ as $s \rightarrow \infty$, for otherwise $\sup _{\pi m=z^{0}} \widehat{g}(m)=0$. For every $\varepsilon>0$ there exists $m_{s_{\varepsilon}}$ with

$$
\left|\widehat{f}\left(m_{0}\right)-\widehat{f}\left(m_{s_{\varepsilon}}\right)\right|<\varepsilon,
$$

which proves that $\widehat{f}\left(m_{0}\right)=1$ so $\pi m_{0}=z^{0}$. Also since $|\widehat{f}| \leq 1$, we have $\left|\widehat{g}\left(m_{s}\right)\right| \geq \sup _{\pi m=z_{0}}|\widehat{g}(m)|$ so, by continuity, $\widehat{g}\left(m_{0}\right)=\sup _{\pi m=z^{0}}|\widehat{g}(m)|$, which proves the proposition.

Theorem 3.3. Suppose $\pi \mathcal{M}=\bar{\Omega}, \pi^{-1}(\Omega)$ is trivial and for every $\xi \in$ $\partial \Omega, \pi^{-1}(\xi)$ is a peak set. If $\mathcal{M} \backslash \overline{\widetilde{\Omega}}$ is separable, then $\mathcal{M} \backslash \overline{\widetilde{\Omega}}$ is contained in a peak set.

Proof. Assume $\left\{m_{j}\right\}_{j=1}^{\infty}$ is dense in $\mathcal{M} \backslash \overline{\widetilde{\Omega}}$. By the proof of a theorem of Forelli [2], we can find $f_{j} \in H^{\infty}(\Omega)$ with $\operatorname{Re} f_{j} \geq 0,\left.\operatorname{Re} \widehat{f}_{j}\right|_{\pi^{-1}\left\{m_{1}, \ldots, m_{j}\right\}} \geq j$, $\operatorname{Re} f_{j}(0) \leq 1 / j^{2}$ and $\operatorname{Im} f_{j}(0)=0$. Define

$$
F_{p}(z)=\frac{\sum_{j=1}^{p} f_{j}}{1+\sum_{j=1}^{p} f_{j}} .
$$


Then $\left|F_{p}(z)\right|<1$ so we can select a subsequence $p_{K}$ such that $F_{p_{K}} \rightarrow f$ uniformly on compact subsets of $\Omega$. Now

$$
|f(0)|=\left|\frac{\sum f_{j}(0)}{1+\sum f_{j}(0)}\right|<1 .
$$

If $m^{0} \in X$ with $z^{0}=\pi\left(m^{0}\right)=\pi\left(m_{j}\right)$, then for a given $p^{0}$ there is a sequence $\left(z^{j}\right)$ in $\Omega$ with $z^{j} \rightarrow z^{0}$ so that $f\left(z^{j}\right) \rightarrow \widehat{f}\left(m^{0}\right)$ and $f_{p^{0}}\left(z^{s}\right) \rightarrow \widehat{f}_{p^{0}}\left(m^{0}\right)$. Thus

$$
\begin{aligned}
& \left|\widehat{1-f}\left(m^{0}\right)\right|=\left|\lim _{s \rightarrow \infty}\left(1-f\left(z^{s}\right)\right)\right|=\left|\lim _{s \rightarrow \infty} \lim _{k \rightarrow \infty} \frac{1}{1+\sum_{j=1}^{p_{k}} \widehat{f}\left(z^{s}\right)}\right| \\
& \leq \lim _{s \rightarrow \infty} \frac{1}{1+\operatorname{Re} f_{p^{0}}\left(z^{s}\right)}=\frac{1}{1+\operatorname{Re} \widehat{f}_{p^{0}}\left(m^{0}\right)} \leq \frac{1}{1+p^{0}} \rightarrow 0, \quad p^{0} \rightarrow \infty .
\end{aligned}
$$

Therefore $\widehat{1-f}\left(m^{0}\right)=0$ so $\widehat{f}=1$ on $\left\{m \in X: \pi m \in \pi\left(\left\{m_{j}\right\}_{j=1}^{\infty}\right)\right\}$. It follows from Proposition 3.2 that $\widehat{1-f}=0$ on $\left\{m \in \mathcal{M}: \pi m=\pi\left(m_{j}\right)\right\}$, $j \in \mathbb{N}$, and since $\left(m_{j}\right)_{j=1}^{\infty}$ is dense in $\mathcal{M} \backslash \overline{\widetilde{\Omega}}, \widehat{f}=1$ on a closed set containing $\mathcal{M} \backslash \overline{\widetilde{\Omega}}$, which completes the proof.

REMARK 1. It follows from the proof that $X=\overline{\widetilde{\Omega}} \backslash \widetilde{\Omega}$ is not separable. For if it were, then we could construct $f$ as in the proof of Theorem 3.3 with $|f(0)|<1$ and $\widehat{1-f}=0$ on $\overline{\widetilde{\Omega}} \backslash \widetilde{\Omega}$. Since $\widetilde{\widetilde{\Omega}} \backslash \widetilde{\Omega}$ contains the Shilov boundary of $H^{\infty}(\Omega)$ it follows that $\widehat{f} \equiv 1$ on $\mathcal{M}$, which is a contradiction.

It follows from the general theory of Banach algebras that if $m_{1} \in \mathcal{M}$ and $f \in H^{\infty}(\Omega)$ are given, then there is an $m_{0} \in \overline{\widetilde{\Omega}}$ such that $\widehat{f}\left(m_{1}\right)=\widehat{f}\left(m_{0}\right)$. If $\left\{m \in \mathcal{M}: \pi m=\pi\left(m_{1}\right)\right\}$ is a peak set, it follows from the Hahn-Banach theorem and Proposition 3.2 that there exists a probability measure with support in $\left\{m \in X: \pi m=\pi\left(m_{1}\right)\right\}$ that represents $m_{1}$.

If we put more conditions on $\Omega$, the next theorem shows that we can always find $m_{0} \in X$ with $\widehat{f}\left(m_{0}\right)=\widehat{f}\left(m_{1}\right)$. It is with great pleasure we acknowledge a discussion with John Erik Fornæss, who suggested how to find a proof of Theorem 3.4.

TheOrem 3.4. Let $\Omega$ be a bounded domain in $\mathbb{C}^{n}$ with the property that for every $\bar{\partial}$-closed form $\lambda \in L_{(0,1)}^{\infty}(\Omega)$ there exists a function $u \in L^{\infty}(\Omega)$ such that $\bar{\partial} u=\lambda$. Let $f \in H^{\infty}(\Omega)$. If $\xi \in \partial \Omega$ such that $\{m \in \mathcal{M}: \pi m=\xi\}$ is a peak set, then for every $m \in \mathcal{M}$ with $\pi m=\xi$ there exists $m_{0} \in \overline{\widetilde{\Omega}}$ such that $\widehat{f}(m)=\widehat{f}\left(m_{0}\right)$ and $\pi m=\pi m_{0}$.

Proof. Let $m \in \mathcal{M}$ with $\pi m=\xi$ be given. We wish to find $m_{0} \in X$. We can assume $\widehat{f}(m)=0$. If there is a sequence $z_{j} \in \Omega$ such that $z_{j} \rightarrow \xi$ and $f\left(z_{j}\right) \rightarrow 0$ as $j \rightarrow \infty$, we have proved the theorem. 
Assume that there are $r, \delta>0$ so that if $z \in \Omega \cap B(\pi m, r)$ then $|f(z)|>\delta$. We have assumed that $\pi m \in \partial \Omega$ and that there exists $g_{0} \in H^{\infty}(\Omega)$ such that $\widehat{g}_{0}(s)=1$ on $\{s \in \mathcal{M}: \pi(s)=\pi(m)\}$ and $\left|\widehat{g}_{0}\right|<1$ otherwise. Define $g=1-g_{0}$ and choose $\chi \in C_{0}^{\infty}(B(\pi m, r / 2))$ with $\chi=1$ near $\pi m$. Then $\bar{\partial} \chi / f g$ is a $\bar{\partial}$-closed $(0,1)$-form with coefficients in $L^{\infty}(\Omega)$. By assumption, there is an $l \in L^{\infty}(\Omega)$ such that $\bar{\partial} l=\frac{\bar{\partial}}{f g} \chi$. Define

$$
\begin{aligned}
& g_{1}=\frac{\chi}{f}-l g \in L^{\infty}, \\
& g_{2}=\frac{1-\chi}{g}+l f \in L^{\infty} .
\end{aligned}
$$

Then

$$
\begin{aligned}
& \bar{\partial} g_{1}=\frac{1}{f} \bar{\partial} \chi-g \bar{\partial} l=0 \\
& \bar{\partial} g_{2}=\frac{-\bar{\partial} \chi}{g}+f \bar{\partial} l=0
\end{aligned}
$$

Furthermore $f g_{1}+g g_{2}=1$ so $0=m 1=1$, which is a contradiction and completes the proof.

REMARK 2. The assumptions of the theorem hold at every boundary point of a strictly pseudoconvex domain.

REMARK 3. The assumptions of the theorem are not necessary for the conclusion to hold. An example is the bidisc $D \times D$. For the homomorphism that projects on the Shilov boundary all the assumptions are satisfied. For the homomorphism $m_{0}$ that projects on the boundary but not on the Shilov boundary the corresponding fiber is not a peak set. But the point evaluations are dense at those points. For if $f \in H^{\infty}(D \times D), \pi m_{0}=(u, v), u \in \partial D$, $v \in D$, then $f(z, w)=f(z, v)+g(z, w)(w-v), g \in H^{\infty}(D \times D)$. Hence $m_{0} f=m_{0} f(z, v)$ so it follows from the corona theorem in $D$ that $m_{0}$ is in the closure of the point evaluations.

Corollary 3.5. Let $\Omega$ be a bounded domain in $\mathbb{C}^{n}$ with the property that for every $\bar{\partial}$-closed form $\lambda \in L_{(0, q)}^{\infty}(\Omega), 1 \leq q \leq n-1$, there exists a form $u \in L_{(0, q-1)}^{\infty}(\Omega)$ such that $\bar{\partial} u=\lambda$. If $\{m \in \mathcal{M}: \pi m=\xi\}$ is a peak set for every boundary point $\xi \in \partial \Omega$, then the following weak form of the corona problem can be solved:

If $f_{1}, \ldots, f_{p-1} \in A(\Omega), f_{p} \in H^{\infty}(\Omega)$ and $\sum_{j=1}^{p}\left|f_{j}\right| \geq \delta>0$ then there exist $g_{1}, \ldots, g_{p} \in H^{\infty}(\Omega)$ with $\sum_{j=1}^{p} g_{j} f_{j} \equiv 1$.

Proof. It is enough to prove that if $m \in \mathcal{M}$ then $\left(m\left(f_{1}\right), \ldots, m\left(f_{p}\right)\right)$ is different from zero. It follows from Proposition 2.2 and the "corona condition" that this is true if $\pi m \in \Omega$. If $\pi m \in \partial \Omega$, it follows from Theorem 3.4 that there is an $m_{1} \in X$ such that $\pi m=\pi m_{1}$ and $m\left(f_{p}\right)=m_{1}\left(f_{p}\right)$. Note 
that, again by Theorem 3.4, $r\left(f_{s}\right)=f_{s}(\pi r)$ for all $r \in \mathcal{M}$ and $1 \leq s \leq p-1$, since $f_{s} \in C(\bar{\Omega})$. Therefore, $\left(m\left(f_{1}\right), \ldots, m\left(f_{p}\right)\right)$ is different from zero in this case too. This completes the proof since $\pi \mathcal{M}=\bar{\Omega}$ by Proposition 2.3.

REMARK 4. Examples of domains with the property that for every $\bar{\partial}$ closed form $\lambda \in L_{(0, q)}^{\infty}(\Omega), 1 \leq q \leq n-1$, there exists a form $u \in L_{(0, q-1)}^{\infty}(\Omega)$ such that $\bar{\partial} u=\lambda$, are strictly pseudoconvex domains ([3]) and analytical polyhedra ([4]).

\section{References}

[1] U. Cegrell, Peak sets and representing measures in the spectrum of $H^{\infty}(\Omega)$, in: Several Complex Variables: Proceedings of the Mittag-Leffler Institut 1987-1988, J.-E. Fornæss (ed.), Math. Notes 38, Princeton Univ. Press, 1993, 48-97.

[2] F. Forelli, Analytic measures, Pacific J. Math. 13 (1963), 571-578.

[3] N. Øvrelid, Integral representation formulas and $L^{p}$-estimates for the $\bar{\partial}$-equation, Math. Scand. 29 (1971), 137-160.

[4] P. L. Polyakov and G. M. Henkin, Integral formulas for solution of the $\bar{\partial}$-equation, and interpolation problems in analytic polyhedra, Trudy Moskov. Mat. Obshch. 53 (1990), 130-170, 260-261 (in Russian).

Department of Mathematics and Mathematical Statistics

Umeå University

S-901 87 Umeå, Sweden

E-mail: linus.carlsson@math.umu.se urban.cegrell@math.umu.se anders.fallstrom@math.umu.se

Received 1.4.2006

and in final form 5.11.2006 